\title{
UNIQUENESS, STABILITY AND ERROR ESTIMATION
}

\author{
BY RAYMOND M. REDHEFFER
}

Communicated by Lipman Bers, March 28, 1963

As in the previous note [1] $B$ is a bounded region, $x=\left(x_{i}\right) \in B$, and subscripts on $u, v, w, c$ denote partial differentiation. We assume $u, v, w, c \in C^{(2)}$ in $B$ and continuous in $\bar{B}$, although these conditions are unnecessarily restrictive. ${ }^{1}$ The normal derivative $u_{\nu}$ is as in [1], $p=+$ or $p=-, \epsilon^{p}$ and $\delta^{p}$ are nonnegative constants, and \|\| denotes the Euclidean norm. Conditions with $T$ are for $x \in B$, those with $R$ for $x \in \partial B$.

As an introduction to the problems we shall consider, let

$$
T u=a(x) u+\sum a_{i}(x) u_{i}-\sum a_{i j}(x) u_{i j}, \quad R u=u-k(x) u_{v}
$$

where $a(x) \geqq A,\left|a_{1}(x)\right| \leqq A_{1}, a_{11}(x)=1,\left[a_{i j}(x)\right] \geqq 0,0 \leqq k(x) \leqq K$, and $\left|x_{1}\right| \leqq b$. Suppose $y=y\left(x_{1}\right)$ satisfies, for $\left|x_{1}\right| \leqq b$ :

$$
E=\inf \left(A y-A_{1}\left|y^{\prime}\right|-y^{\prime \prime}\right)>0, \quad D=\inf \left(y-K\left|y^{\prime}\right|\right)>0 .
$$

Then the inequalities

$$
p(T u-T v) \leqq \epsilon^{p} \text { and } p(R u-R v) \leqq \delta^{p}
$$

imply $p(u-v) \leqq y\left(x_{1}\right) \max \left(\epsilon^{p} / E, \delta^{p} / D\right)$. The condition on $a_{1}$ can be replaced by $a_{1} \geqq-A_{1}$ if $y^{\prime} \geqq 0$ and by $a_{1} \leqq A_{1}$ if $y^{\prime} \leqq 0$.

The usefulness of this result and the ease of its proof suggest that it be extended to nonlinear problems, and that is our purpose. We first consider the operator and continuity conditions:

$$
\begin{aligned}
T u & =a\left(x, u, u_{i}\right)-\sum a_{i j}\left(x, u_{k}\right) u_{i j}, \\
\left\|a_{i j}\left(x, u_{k}\right)-a_{i j}\left(x, v_{k}\right)\right\| & \leqq A_{1}\left\|u_{k}-v_{k}\right\|, \\
p\left[a\left(x, u, u_{i}\right)-a\left(x, v, v_{i}\right)\right] & \geqq-A_{1}\left\|u_{i}-v_{i}\right\| \text { for } p(u-v)>0 .
\end{aligned}
$$

Usually these hold only for $u$ and $v$, but in Theorem 1 they hold for every pair of solutions, the constants depending on the pair:

THEOREM 1. Let $T$ be as in (2) and let $R u=k_{1}(x, u)-k_{2}\left(x, u_{v}\right)$ whore $k_{2}$ is nondecreasing and $k_{1}$ is strictly increasing in the last argument. Suppose the problem $T w=\bar{\tau}(x), R w=\rho(x)$ has solutions of the following two types:

${ }_{1}^{1}$ The main condition really needed is that $p(u-v)$ be upper semicontinuous in the closure of the set where $p(u-v)>0$. 
(i) a solution $w=u$ such that $\left[a_{i j}\left(x, u_{k}\right)\right] \geqq 0, x \in B$;

(ii) a solution $w=v$ such that, for some function $c(x)$,

$$
\inf _{x} \sum a_{i j}\left(x, v_{k}\right) c_{i} c_{j}>0, \quad \inf _{x} \sum a_{i j}\left(x, v_{k}\right) c_{i j}>-\infty, \quad x \in B .
$$

Then $u=v$, and there is no other solution.

The hypothesis (i) can be written in the form

$$
m_{1}(x)\left[a_{i j}\left(x, u_{k}\right)\right]+m_{2}(x)\left[a_{i j}\left(x, v_{k}\right)\right] \geqq 0
$$

and the hypothesis (ii) in the form

$$
\begin{gathered}
\sum m_{i j} c_{i} c_{j} \geqq \mu_{1}, \quad \sum m_{i j} c_{i j} \geqq \mu_{2}, \\
m_{i j}=m_{3}(x) a_{i j}\left(x, u_{k}\right)+m_{4}(x) a_{i j}\left(x, v_{k}\right) .
\end{gathered}
$$

We now suppose the $m_{i}$ are any functions whatever satisfying

(3c) $m_{1}+m_{2}=m_{3}+m_{4}=1, \quad\left|m_{i}\right| \leqq M_{i}, \quad\left|m_{1}-m_{3}\right| \leqq M_{13}$.

A hypothesis $\mathrm{H}$ is said to hold "in the neighborhood of the maximum" if to each fixed compact subset $S \subset B$ corresponds a positive constant, $\eta$, such that $\mathrm{H}$ holds at those points of $S$ where simultaneously

$$
\left\|u_{i}-v_{i}\right\|<\eta, \quad m-\eta<p(u-v)<m, \quad m=\sup p(u-v) .
$$

THEOREM 2. Let $R$ be as in Theorem 1, and let the following hypothesis hold in the neighborhood of the maximum: T has the form (2), and there exist multipliers $m_{i}$ and a function $c$ such that (3) holds with $\mu_{1}>0, \mu_{2}>-\infty$. Then

$$
p(T u-T v) \leqq 0 \quad \text { and } \quad p(R u-R v) \leqq 0 \Rightarrow p(u-v) \leqq 0 .
$$

This result (which contains Theorem 1 ) follows by setting $y=\alpha_{0}$ $-\alpha e^{\beta c}$ and considering the behavior of $p(u-v)-y$ at its maximum. A similar method yields stability when

$$
\begin{aligned}
R u=u-k\left(x, u_{\nu}\right), p\left[k\left(x, u_{\nu}\right)-k\left(x, v_{v}\right)\right] \leqq & K(|s|) \\
& \text { for } p\left(u_{\nu}-v_{v}\right) \leqq s,
\end{aligned}
$$

the function $K(s)$ being continuous, nonnegative, and increasing for $s \geqq 0$. Assuming

(5) $\quad\left\|u_{i j}\right\| \leqq U_{2}, \quad\left\|v_{i j}\right\| \leqq V_{2}, \quad 0 \leqq c \leqq C, \quad\left\|c_{i}\right\| \leqq 1, \quad\left\|c_{i j}\right\| \leqq C_{2}$

we have:

Theorem 3. Let (1)-(5) hold with $\mu_{1}>0$, let $\lambda>0$, and let $\mu_{1} \beta=1$ or

$$
\mu_{1} \beta=1+A_{1}+A_{2}\left(M_{2} U_{2}+M_{1} V_{2}\right)+\lambda A_{2} M_{13}-\mu_{2},
$$


whichever is larger. Let $\epsilon^{p} \leqq \epsilon_{0}$, where $\epsilon_{0}\left(\beta+C_{2}\right)=\lambda$ sech $\beta C$, and define $\xi^{p}=\epsilon^{p} \sinh \beta C$. Then $p(u-v) \leqq \delta^{p}+C \xi^{p}+K\left(\xi^{p}\right)$.

If $\left(c_{i j}\right) \geqq 0$ we can replace $C_{2}$ and $\mu_{2}$ by 0 in Theorem 3 .

Similar results are valid for the operator

(6a) $T u=a\left(x, u, u_{i}\right)-\sum a_{i j}\left(x, u_{k}\right) u_{i j}-\sum a_{i j k l}\left(x, u_{m}\right) u_{i j} u_{k l}$

where $u_{m}$, like $u_{i}$ and $u_{k}$, means $\left(u_{1}, u_{2}, \cdots, u_{n}\right)$. Denoting the function on the right of $(6 \mathrm{a})$ by $-f$, we set

$$
\begin{aligned}
f_{i j}(u) & =a_{i j}\left(x, u_{k}\right)+\sum\left[a_{i j k l}\left(x, u_{m}\right)+a_{k l i j}\left(x, u_{m}\right)\right] u_{k l}, \\
M_{i j} & =f_{i j}(u)+f_{i j}(v) .
\end{aligned}
$$

TheOREM 4. Let $T$ be as in (6), let $R u=k\left(x, u, u_{\nu}\right)$, and let $\alpha$ and $\beta$ be nonnegative constants. Suppose $\left(M_{i j}\right) \geqq 0$ for $u_{i}=v_{i}$, and further:

(i) $p\left[a\left(x, u, u_{i}\right)-a\left(x, v, v_{i}\right)\right]>\epsilon^{p} \quad$ for $p(u-v)>\alpha, \quad u_{i}=v_{i}$,

(ii) $p\left[k\left(x, u, u_{\nu}\right)-k\left(x, v, v_{\nu}\right)\right]>\delta^{p} \quad$ far $p(u-v)>\beta, p u_{v} \leqq p v_{\nu}$.

Then (1) implies $p(u-v) \leqq \max (\alpha, \beta)$.

The proof follows by consideration of $p(u-v)-y, y=\max (\alpha, \beta)$. We now assume

$$
\left\|a_{i j k l}\left(x, u_{m}\right)-a_{i j k l}\left(x, v_{m}\right)\right\| \leqq B_{2}\left\|u_{m}-v_{m}\right\| .
$$

The procedure used for Theorem 2 yields:

Theorem 5. Let $T$ be as in (6) and $R$ as in Theorem 1. Suppose the following hold in the neighborhood of the maximum:

(i) for some $c(x)$ the matrix $\left(M_{i j}\right)$ satisfies

$$
\left(M_{i j}\right) \geqq 0, \quad \inf _{x} \sum M_{i j} c_{i} c_{j}>0, \quad \inf _{x} \sum M_{i j} c_{i j}>-\infty,
$$

(ii) the continuity conditions (2) and (7) are valid.

Then $p(T u-T v) \leqq 0$ and $p(R u-R v) \leqq 0 \Rightarrow p(u-v) \leqq 0$.

If the coefficients in (6) do not involve the first derivatives then (i) can be replaced by $\left(i^{\prime}\right):$ The matrix $\left(M_{i j}\right) \geqq 0$ and has only countably many zeros in $B$. This extension holds if, instead of $u, v \in C^{(2)}$, we have only existence of $(u-v)_{i}$ at each $x$ for some $i=i(x)$. Theorem 5 implies a uniqueness theorem that strongly generalizes the results of Rellich [3].

As in [2] the Lipschitz-type terms $A\left\|u_{i}-v_{i}\right\|$ in Theorems 1, 2 and 5 can be replaced by $g\left(\left\|u_{i}-v_{i}\right\|\right)$ where the function $g(s)$ is positive, continuous and increasing for $s>0$, and $\int_{0}^{1} d s / g(s)=\infty$. By requiring 
$c_{\nu}>0$ and strict monotony of $k_{2}\left(x, u_{\nu} \uparrow\right)$ on part of $\partial B$ one readily extends these results to mixed boundary-value problems. Corners are allowed, even if $R u=-u_{\nu}$ at all but one point of $\partial B$.

\section{BiBLIOGRAPHY}

1. R. M. Redheffer, Differential inequalities, Bull. Amer. Math. Soc. 69 (1963), 239-242.

2. - An extension of certain maximum principles, Monatsh. Math. 66 (1962), $32-42$.

3. F. Rellich, Zur ersten Randwertaufgabe bei Monge-Ampèreschen Differentialgleichungen vom elliptischen Typus, Math. Ann. 107 (1933), 505-513.

University of California, Los Angeles 